\title{
Fracture analysis of anodically bonded silicon substrates during the CMP process
}

\author{
Sung-min Sim ${ }^{1}$, Yeonsu Lee ${ }^{1}$, Hye-Lim Kang ${ }^{1}$, Youngsuk Hwang ${ }^{2}$, Chi-Hyun Park ${ }^{2}$, Ignacio Llamas-Garro ${ }^{3}$ \\ and Jung-Mu Kim ${ }^{4^{*}}$
}

\begin{abstract}
In this paper, a stress and fracture study, occurring during the chemical mechanical polishing (CMP) of anodically bonded substrates is presented. The samples contain glass pillars, used to form the glass cavities and a silicon substrate sealing the glass structure, the samples are fabricated using the anodic bonding process. The mechanical stresses of the bonded silicon substrate are simulated using the COMSOL software. The fracture strength after post-processing is investigated based on the criterion value, which is the ratio of the anodically bonded area over the cavity area. It is found that the bonded area and the distribution of pillars are related to the mechanical stability of the bonded substrate during the CMP process. Studies on the stability of subsequent processes, like CMP after anodic bonding, plays an important role in improving the fabrication yield of anodic bonded devices.
\end{abstract}

Keywords: Anodic bonding, Chemical mechanical polishing, von Mises stress

\section{Introduction}

Wafer bonding is used in microelectromechanical systems (MEMS) to protect and package sensitive internal structures from environmental influences, such as temperature, humidity and pressure. Wafer bonding is classified into direct bonding, anodic bonding and bonding performed by introducing an intermediate layer [1]. The anodic bonding process is among the wafer bonding techniques widely used for MEMS packaging, since it provides strong bonding strength, hermetic encapsulation, high temperature resistance and permanent bonding; frequently used to package devices such as accelerometers and pressure sensors $[2,3]$.

Anodic bonding should be done under an electric field at high temperatures. When temperature and voltage are applied after the silicon and glass substrates are in contact, they are bonded by covalent bonds, formed at the bonding interface between the silicon and glass. In the process, both substrates have typical requirements for a successful bond, involving low surface roughness

\footnotetext{
*Correspondence: jungmukim@jbnu.ac.kr

${ }^{4}$ Department of Electronic Engineering, Chonbuk National University, Jeonju 54896, Republic of Korea

Full list of author information is available at the end of the article
}

$(<10 \mathrm{~nm})$. A temperature lower than the glass transition temperature is applied to increase the glass ion mobility. The applied temperature and voltage induce glass chemical bond dissociation, the cations $\left(\mathrm{Na}^{+}\right)$in the dissociated ions drift toward the backside of the glass, where the cathode is applied. As the cations move, the anions $\left(\mathrm{O}^{2-}\right)$ in the dissociated ions remain at the bonding interface. Then, a depletion region is formed by the applied voltage, resulting in an irreversible bond. In other words, the remaining anions $\left(\mathrm{O}^{2-}\right)$ react with the silicon surface to form silicon oxide, resulting in two anodically bonded substrates.

After the anodic bonding process, the bonded substrates usually become the final electronic device, through performing subsequent processes such as chemical mechanical polishing (CMP), thin film deposition, etch and dicing $[4,5]$. Subsequent processes are indispensable for the complete fabrication of a specific MEMS device; however fracture is frequently observed on the anodically bonded substrates, caused during the subsequent processes [5-7]. Since the two materials (glass and silicon) have slightly different thermal expansion coefficients, the substrates bonded at high temperatures will have a residual stress at room temperature, resulting in fractures on the bonded substrate, obtained 
during the subsequent processes. In other words, the anodically bonded substrate involving residual stress may be fractured or deformed, due to mechanical and chemical influences present during the subsequent processes. Therefore, stress analysis is important to improve subsequent process stability and device production yield.

In this paper, the stability and fabrication yield regarding the subsequent processes can be improved by studying the fractures occurring on anodically bonded substrates, with respect to the area and shape of the bonding interface. A stress and fracture model of Microinfinity Co., Ltd., company that manufactures and commercializes MEMS devices, was simulated using the COMSOL software. The stresses present on the anodically bonded substrates were analyzed based on the ratio of bonded area to cavity area. The results allow to predict the deformation and fracture possibility during the CMP process, after anodic bonding.

\section{Design and fabrication}

Figure 1 shows a schematic diagram of the bonded substrates, where the glass substrate contains the cavities, the glass substrate is sealed with a silicon substrate by using the anodic bonding process. The bonded substrates are designed and fabricated by Microinfinity Co., Ltd. for commercial resonator and accelerometer production.

In order to analyze the stresses of the bonded substrates with respect to area and shape of the bonded interface between glass and silicon substrates, the glass substrate has four cavity patterns, designed as shown in Fig. 2. The bonding area rate (BAR) is used in each sample for the von Mises stress analysis, present during the CMP process:

$$
\mathrm{BAR}=\frac{\text { Anodic bonded area }}{\text { Total area of cavity }} .
$$

The glass substrate cavity area is $6.37 \times 6.37 \mathrm{~mm}^{2}$, and the cavity depth is $30 \mu \mathrm{m}$. The anodically bonded area of sample 1 is $2.8 \mathrm{~mm}^{2}$, and the areas of samples 2,3 , and 4 are $3.1 \mathrm{~mm}^{2}, 3.5 \mathrm{~mm}^{2}$, and $8.6 \mathrm{~mm}^{2}$, respectively. All samples have an internal structure for the MEMS sensor in the cavity (see Fig. 2). Sample 1 with a BAR of $7.0 \%$

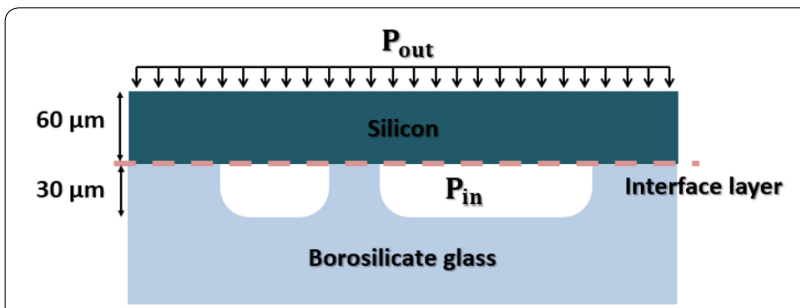

Fig. 1 Schematic diagram of the anodically bonded substrate has only the internal structures for the MEMS sensor in the cavity, these are also used to support the sealed cavity. Glass pillars with a diameter of $20 \mu \mathrm{m}$ are arranged in the cavity at $320 \mu \mathrm{m}$-intervals in sample 2 , with a BAR of $7.6 \%$. A glass pillar array with a pitch of about $1.2 \mathrm{~mm}$ is arranged to support the cavity space in sample 3 , with a BAR of $8.5 \%$. For sample 4, glass pillars with a radius of $15 \mu \mathrm{m}$ are arranged at intervals of $60 \mu \mathrm{m}$, with a BAR of $21.2 \%$.

Figure 3 shows the fabrication process used to create the samples, where anodic bonding and CMP processes are performed. A borosilicate glass substrate having a thermal expansion coefficient of $3.3 \times 10^{-6} / \mathrm{K}$, similar to the silicon substrate $\left(2.6 \times 10^{-6} / \mathrm{K}\right)$ was used. A photoresist mask was patterned on the glass substrate to form the cavities on desired locations and areas of the substrate. Using an HF-based etchant, the unmasked regions were engraved as cavities. Both silicon and glass substrates are cleaned to reduce surface roughness. The anodic bonding process took place in a vacuum better than $1.33 \mathrm{~Pa}$. The anodically bonded substrates were polished using the CMP process until the thickness of the silicon substrate became $60 \mu \mathrm{m}$. As shown in Table 1, the CMP process was performed at an external pressure $\left(\mathrm{P}_{\text {out }}\right)$ of $5.37 \mathrm{kPa}$ and an internal pressure $\left(\mathrm{P}_{\text {in }}\right)$ of $1.33 \mathrm{~Pa}$ at a temperature of $25^{\circ} \mathrm{C}$.

\section{Results and discussion}

The stress occurring during the CMP process was simulated using the COMSOL Multiphysics ${ }^{\circledR}$ software (COMSOL, Inc., USA). Figure 4 shows the von Mises stress at the bonded interface between the silicon and glass substrates for each sample. The von Mises stress applied to sample 1 is concentrated at the edge of the internal structure for the MEMS sensor. In samples 2 and 4, the von Mises stress is concentrated in the middle part of the bonded interface without glass pillars. In sample 3, the von Mises stress is evenly distributed both on the internal structure and on the glass pillars. Figure 5 shows the maximum von Mises stress for each sample. The maximum von Mises stress of sample 1 is $89.50 \mathrm{MPa}$, which exceeds the fracture stress of borosilicate glass $(69 \mathrm{MPa})$ [8], thus the substrate will be destroyed during the CMP process, after the silicon and glass substrates are anodically bonded. On the other hand, samples 2,3 and 4 have improved stability for subsequent processes, by using a wider bonding area, compared to sample 1. As shown in Fig. 5, the BAR increases from 7.0 to $21.2 \%$, and the maximum von Mises stress decreases from $89.50 \mathrm{MPa}$ to $0.30 \mathrm{MPa}$ as the bonded area between glass and silicon is widened. The BAR of samples 2 and 3 are $7.6 \%$ and $8.6 \%$, respectively, which are slightly larger values than the BAR of sample 1 (7.0\%). However, the maximum von Mises 
$<$ Sample $1>$

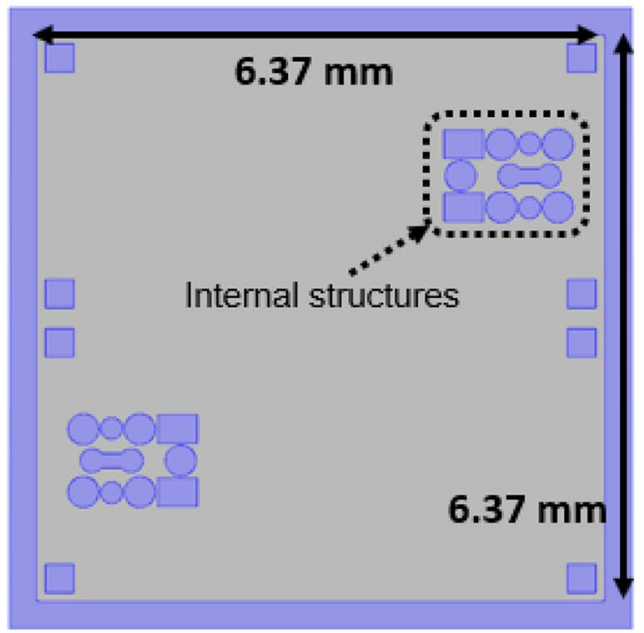

$<$ Sample $3>$

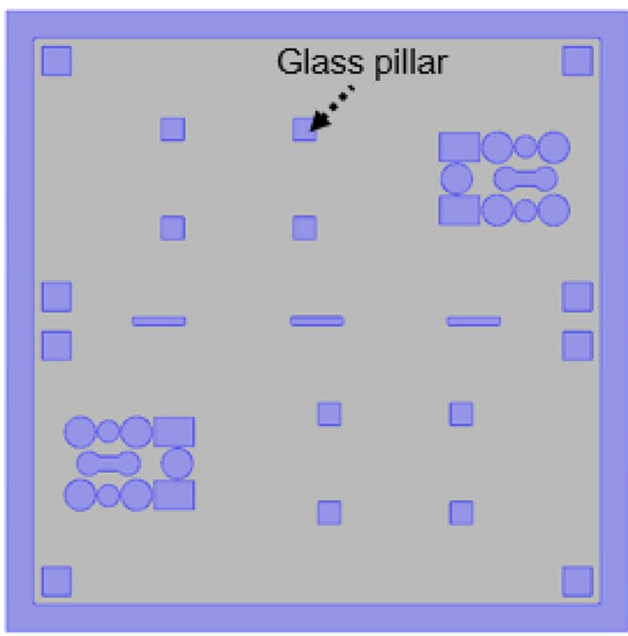

< Sample 2>

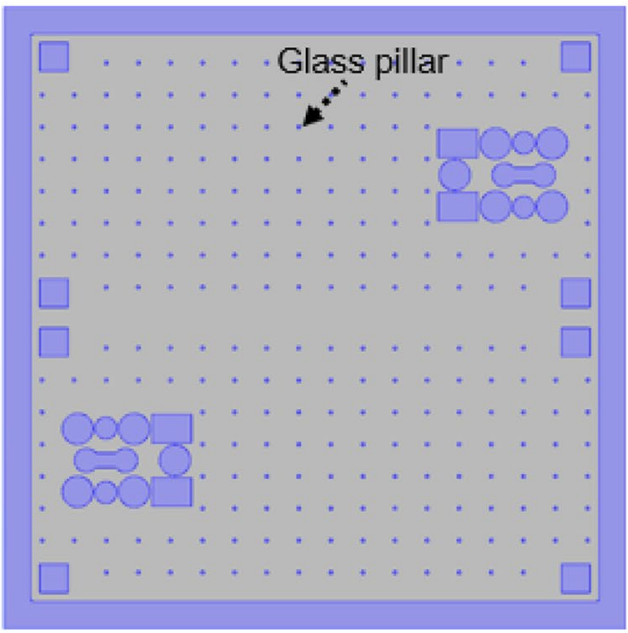

< Sample $4>$

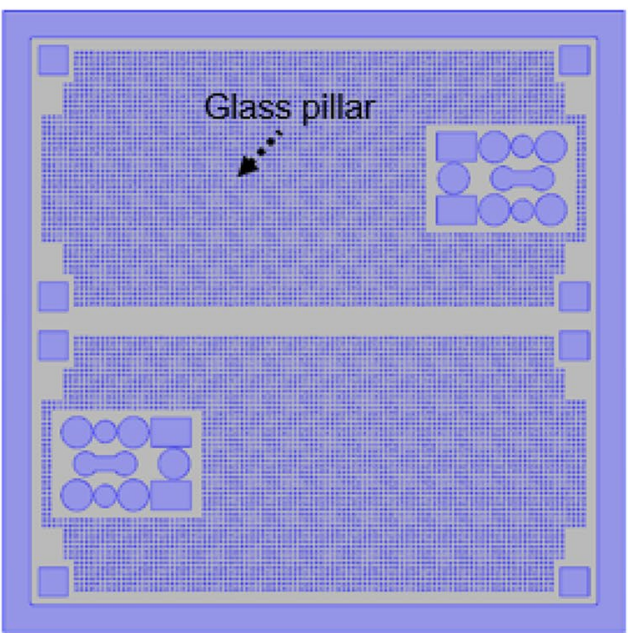

Fig. 2 Anodic bonded sample interface, used to analyze the stress occurring during the CMP process: bonded area (blue color), glass cavity (silver color)

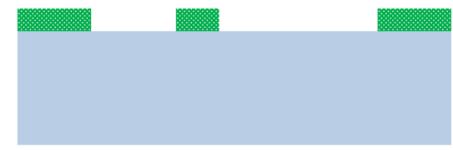

1. PR patterning

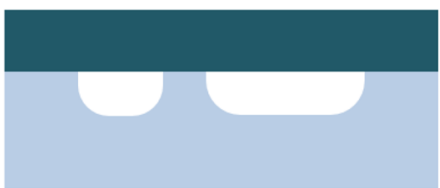

4. Anodic bonding

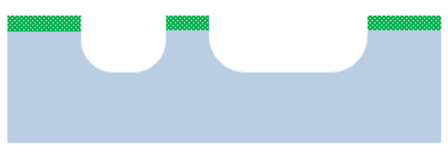

2. HF-based etching

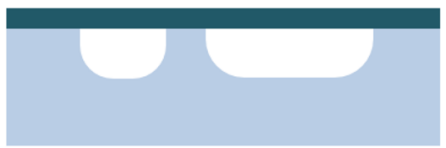

5. CMP process

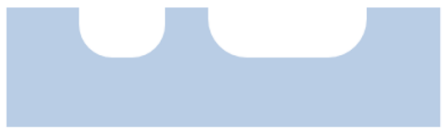

3. PR removal : glass : : photoresist : $\mathrm{Si}$

Fig. 3 Fabrication process of the anodically bonded substrate, and the subsequent CMP process 
stresses of samples 2 and 3 are $1.58 \mathrm{MPa}$ and $9.27 \mathrm{MPa}$, respectively, which is much lower than the maximum von Mises stress of sample $1(89.50 \mathrm{MPa})$. For the samples, the glass pillars are arranged in the cavity. The presence of these glass pillars greatly affects the stress reduction. Figure 6 shows the SEM image and the optical microscope image of the fabricated samples 1 and 3 . As shown in Fig. 4, sample 1 was broken during the CMP process in the region where the von Mises stress is concentrated. On the other hand, sample 3 was stably prepared for the CMP process after the anodic bonding process by

Table 1 CMP process conditions of anodically bonded substrate

\begin{tabular}{|c|c|c|c|c|c|}
\hline $\begin{array}{l}\text { Temperature } \\
\left({ }^{\circ} \mathrm{C}\right)\end{array}$ & $\begin{array}{l}\mathrm{P}_{\text {out }} \\
\text { (kPa) }\end{array}$ & $P_{\text {in }}(\mathrm{Pa})$ & $\begin{array}{l}\text { Silicon } \\
\text { thickness } \\
(\mu \mathrm{m})\end{array}$ & $\begin{array}{l}\text { Glass } \\
\text { cavity } \\
\text { depth } \\
(\mu \mathrm{m})\end{array}$ & $\begin{array}{l}\text { Glass } \\
\text { thickness } \\
(\mu \mathrm{m})\end{array}$ \\
\hline 25 & 5.37 & 1.33 & 60 & 30 & 675 \\
\hline
\end{tabular}

relieving the stress concentration using the glass pillars. When the von Mises stress is concentrated over $69 \mathrm{Mpa}$ during the CMP process, the glass will be destroyed first (see bottom view of Fig. 6). It is believed that the sharp edges and particles of the broken glass destroyed the silicon substrate (see top view of Fig. 6).

The simulation results show that the higher the BAR is, the lower the stress is, during the CMP process. However, if the BARs are similar as in the case of samples 1,2, and 3 , additional factors must be considered during the stress analysis. As mentioned above, sample 2 and 3 significantly reduced the stress by using glass pillars, although the BAR was similar to sample 1 . In samples 2 and 3 , the glass pillars supporting the cavity were arranged at intervals of $0.32 \mu \mathrm{m}$ and $1.2 \mathrm{~mm}$, respectively; the two samples used glass pillars with a similar BAR, but samples 2 and 3 were designed to have narrow and wide spacing arrangements of glass pillars, respectively. Sample 2 has undergone the less stress, because the glass pillars of sample 2 were more densely dispersed compared to sample 3 . In
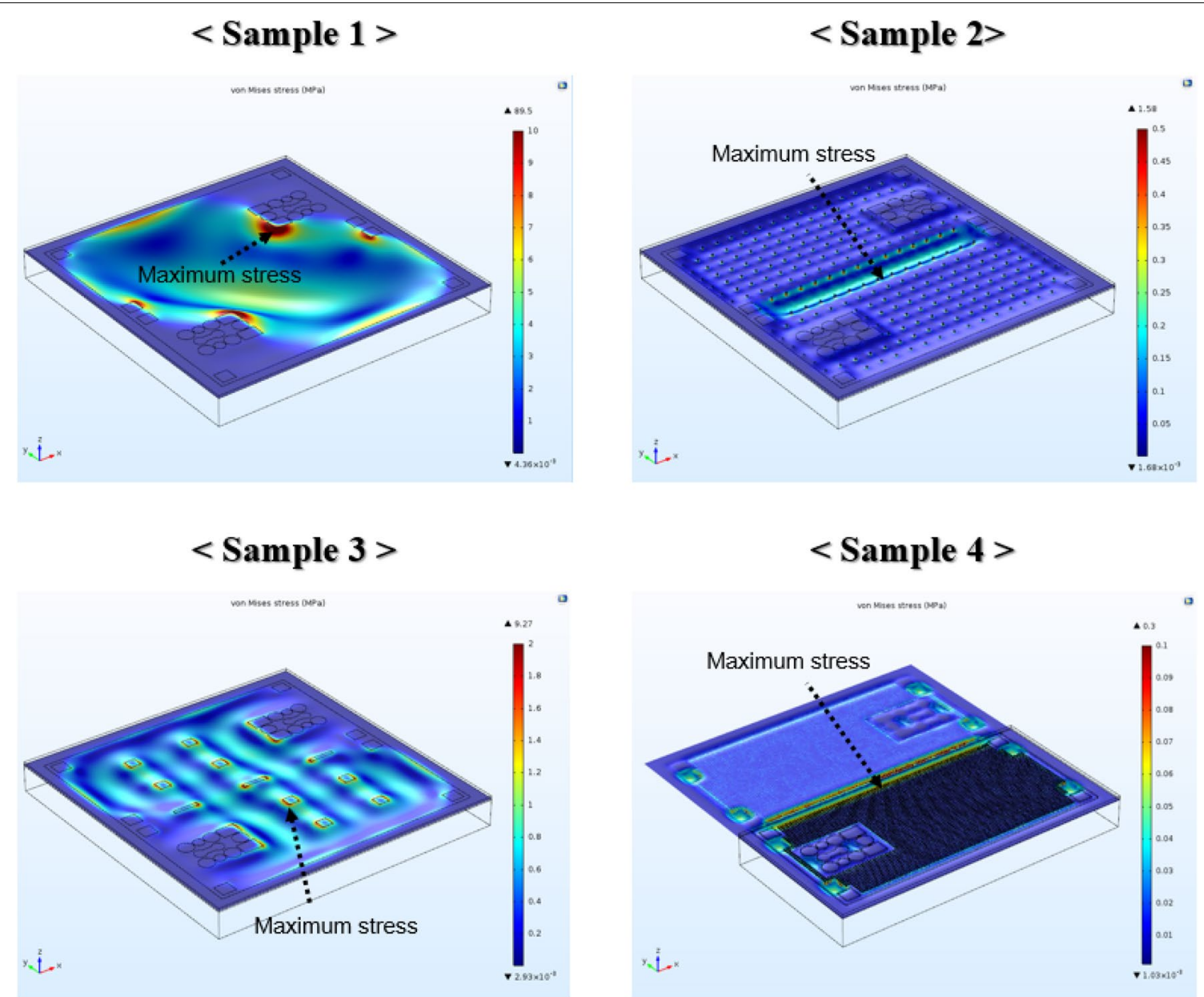

Fig. 4 Von Mises stress distribution map at the bonded interface between the silicon and glass substrates 


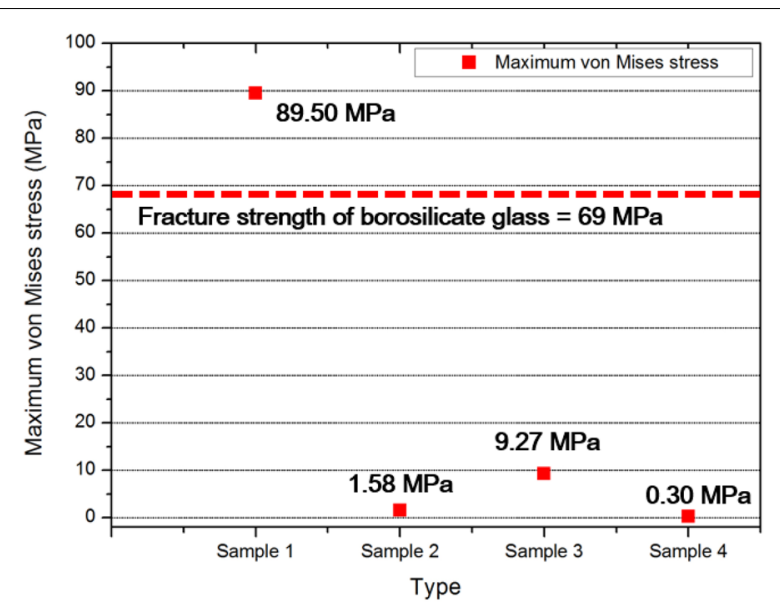

Fig. 5 Maximum von Mises stress for each sample other words, the distribution of glass pillars apparently has a greater impact on stress distribution than the BAR, in the case of structures having a similar BAR. Table 2 shows the comparison of bonding properties for the four types of cavity pattern samples. Consequently, the anodically bonded areas and distribution of the glass pillars play a major role in preventing the fracture of the anodically bonded substrates during the subsequent CMP process.

\section{Conclusion}

Anodically bonded substrates having a large cavity are fabricated for a silicon MEMS device. Anodic bonded substrates with four different bonding areas are simulated for the analysis of stresses, occurring during the CMP process. In order to prevent anodically bonded substrate
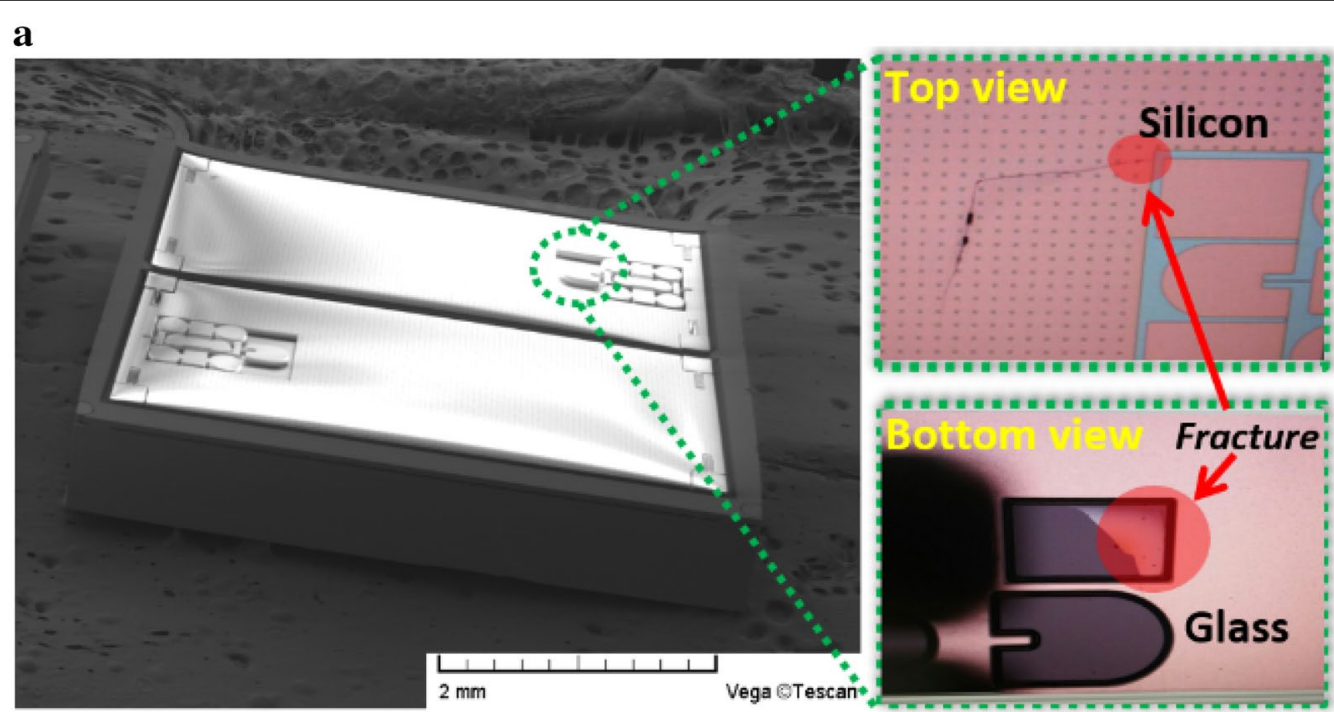

b

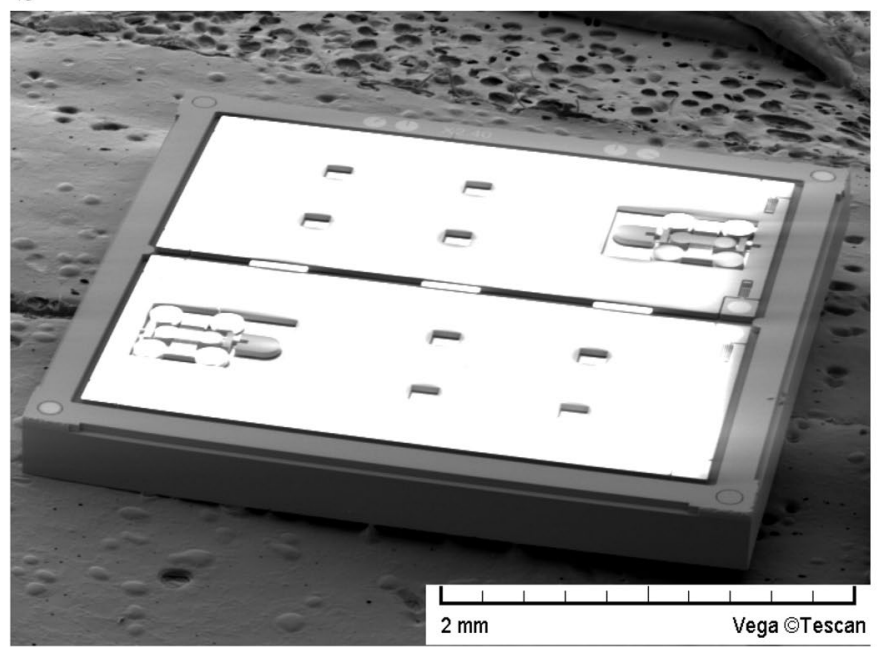

Fig. 6 Anodically bonded substrate fabrication results, a SEM and optical microscope image of the fractured sample 1, b SEM and optical microscope image of the unfractured sample 3 
Table 2 Comparison of bonding properties for the cavity patterns

\begin{tabular}{|c|c|c|c|c|}
\hline & Sample 1 & Sample 2 & Sample 3 & Sample 4 \\
\hline Area of glass pillar $\left(\mathrm{mm}^{2}\right)$ & 2.8 & 3.1 & 3.5 & 8.6 \\
\hline Bonding area rate (\%) & 7.0 & 7.8 & 8.6 & 21.2 \\
\hline Maximum von Mises stress (MPa) & 89.50 & 1.58 & 9.27 & 0.3 \\
\hline Occurrence of fractures & Yes & No & No & No \\
\hline
\end{tabular}

fracture, the stress intensity of four samples is analyzed according to the bonded area and pattern of the glass pillar arrays inside the cavity. As the ratio of bonded area to cavity area increased from 7.0 to $21.2 \%$, the von Mises stress occurring during the CMP process was relieved from 89.5 to $0.3 \mathrm{MPa}$. As a result, the anodically bonded area and the distribution of the glass pillars inside the cavity are important factors for a stable subsequent CMP process, impacting the productivity, reliability and fabrication yield of the MEMS device.

\section{Authors' contributions}

All authors read and approved the final manuscript.

\section{Author details}

${ }^{1}$ Division of Electronics and Information Engineering, Chonbuk National University, Jeonju 54896, Republic of Korea. ${ }^{2}$ Microinfinity Co., Ltd, Suwon 16229, Republic of Korea. ${ }^{3}$ Centre Tecnològic de Telecomunicacions de Catalunya, CERCA, Castelldefels, 08860 Barcelona, Spain. ${ }^{4}$ Department of Electronic Engineering, Chonbuk National University, Jeonju 54896, Republic of Korea.

\section{Acknowledgements}

Not applicable.

\section{Competing interests}

The authors declare that they have no competing interests.

\section{Availability of data and materials}

Not applicable.

\section{Funding}

This work was financially supported by the National Research Foundation of Korea (NRF-2017R1A2B4005687). Part of this work has been supported by the Generalitat de Catalunya under Grant 2017 SGR 891.

\section{Publisher's Note}

Springer Nature remains neutral with regard to jurisdictional claims in published maps and institutional affiliations.
Received: 7 October 2018 Accepted: 4 December 2018

Published online: 07 December 2018

\section{References}

1. Cunningham Shawn, Kupnik Mario (2011) Wafer bonding. In: Ghodssi Reza, Lin Pinyen (eds) MEMS materals and processes handbook. Springer, New York

2. Hinzel DH, Goldsmith CL, Linder LF (2003) Method of integrating MEMS device with low-resistivity silicon substrates. US Patent 6,559,530 B2, May, 2003

3. Seki T, Uno Y, Narise K, Masuda T, Inoue K, Sato S, Sato F, Imanaka K, Sugiyama S (2006) Development of a large-force low-loss metal-contact RF MEMS switch. Sens Actuators A 132(2):683-688

4. Lee Byeungleul, Seok Seonho, Chun Kukjin (2003) A study on wafer level vacuum packaging for MEMS devices. J Micromech Microeng 13(5):663-669

5. Auersperg J, Auerswald E, Collet C, Dean Th, Vogel D, Winkler Th, Rzepka $S$ (2018) Investigations of the impact of initial stresses on fracture and delamination risks of an avionics MEMS pressure sensor. Microelectron Reliab 87:238-244

6. Ma Zhibo, Wang Yinan, Shen Qiang, Zhang Han, Guo Xuetao (2018) Key processes of silicon-on-glass MEMS fabrication technology for gyroscope application. Sensors. 18(4):1240

7. Sim S, Lee Y, Kang H-L, Hwang Y, Park C-H, Llamas-Garro I, Kim J-M (2017) Stress analysis of anodic bonded wafer after CMP process In: Proceedings of 43rd international conference on micro and nano engineering (mne2017), 18-22 September 2017

8. Ring Terry A, Feeney Paul, Boldridge David, Kasthurirangan Jaishankar, Li Shoutian, Dirksen James A (2007) Brittle and ductile fracture mechanics analysis of surface damage caused during CMP. J Electrochem Soc 154(3): $\mathrm{H} 239-\mathrm{H} 248$

\section{Submit your manuscript to a SpringerOpen ${ }^{\circ}$ journal and benefit from:}

- Convenient online submission

$\checkmark$ Rigorous peer review

- Open access: articles freely available online

- High visibility within the field

- Retaining the copyright to your article

Submit your next manuscript at $>$ springeropen.com 